\title{
THE SUPERCONDUCTING MAGNET SYSTEM FOR THE ATLAS DETECTOR AT CERN
}

\author{
Paola Miele and Herman H J ten Kate, on behalf of the ATLAS Collaboration \\ CERN, CH-1211 Geneva 23, Switzerland
}

\begin{abstract}
The ATLAS superconducting magnet system for the ATLAS detector in the LHC at CERN comprises a Barrel Toroid, two End Cap Toroids and a Central Solenoid, with overall dimensions of $20 \mathrm{~m}$ diameter by $26 \mathrm{~m}$ length. Though this magnet system is used for a particle detector, its size, geometrical arrangement and coil technology is comparable to what is usual for fusion machines. The manufacturing has started in the industry and integration of the magnets will start in the year 2001. Assembly in the cavern is planned in the year 2003, starting with BT installation. In this paper the Magnet System is presented as well as the common infrastructure and services.
\end{abstract}

\section{INTRODUCTION}

The superconducting Magnet System for the ATLAS Detector for the Large Hadron Collider at CERN to analyze proton-proton collisions at $14 \mathrm{TeV}$ level has already passed the design phase and is now under construction in industry. The realization of the detector and corresponding infrastructure is organized by the ATLAS Collaboration, a joint enterprise of about 145 institutes in 34 countries world-wide including CERN. The challenge of ATLAS to reach optimum physics performance requires the particular magnetic field configuration made up by the axial field created by the Central Solenoid and the tangential field created by the Toroid System.

The ATLAS Magnet System [1,2] comprises the 4 superconducting magnets, the power supply, the cryogenics, the vacuum and the control and safety systems. The magnet system has overall dimensions of 26 meters in length and 20 meters in diameter, Fig. 1. The superconducting magnets comprise the Toroidal Field (TF) magnet system with the Barrel Toroid (BT) and the 2 End Cap Toroids (ECT) and the Central Solenoid (CS). Each of the 3 toroids consists of 8 coils connected in series, with flat pancake type of windings, assembled around the beam axis with an offset angle of $22.5^{0}$ between the BT and ECT systems, in order to optimize the bending 
power in the available space. A model coil of reduced length of BT, the B0 model coil, is under construction to qualify the manufacturing cycle and related techniques. The Central Solenoid is a single layer coil wound internally in a supporting cylinder and enclosed by the common cryostat of the Liquid Argon Calorimeter. Aluminum stabilized NbTi/Cu superconductor cooled at $4.5 \mathrm{~K}$ by liquid Helium forced flow and glass fibers-epoxy resin insulation are the main components of the coils. A $21 \mathrm{kA}$ common TF power system and an $8 \mathrm{kA}$ CS power system with correspondent quench protection systems are implemented.

The 4 main phases for the realization of the Magnet System comprise design, construction, test and installation. A co-operation between CERN/ATLAS and 5 magnet laboratories is the basis for the design and construction of the ATLAS Magnet System. CEA-Saclay (F) and INFNLASA (I) are responsible for the BT, RAL (UK) and NIKHEF (Nl) for the ECT and KEK (J) for the CS. CERN is mainly responsible for the overall project management and co-ordination, as well as the infrastructure for assembly and testing, the installation underground and the common cryogenics, power, vacuum, control and safety systems.

The main parameters of the magnets are listed in Table 1.

\section{CONDUCTOR AND WINDING}

The superconductor used is a composite consisting of a flat $\mathrm{NbTi} / \mathrm{Cu}$ Rutherford cable coextruded with aluminium stabiliser with rectangular cross section. For the BT and ECT the stabiliser is high purity aluminium while in the case of the CS high-strength aluminium was developed by KEK. The production of the conductor for the CS is completed by Furukawa $(6 \mathrm{~km})$ and Hitachi $(6 \mathrm{~km})$, while it is planned to be completed by the end of the year 2000 for the BT and ECT by Vacuumschmelze (D) and LMI (I)-ACS/ETH (CH).

Table 1 shows the main characteristics of the conductors.

The qualification of the conductor is fully established, as well as reproducibility and reliability in long unit lengths. Crucial for acceptance of produced conductor units is the critical current, the aluminium RRR and the bond quality between aluminium and copper. This to fulfil 
the requirements of high mechanical shear strength and low electrical and heat transfer resistance, which guarantee proper cooling, stability and protection against conductor burn-out. The coils are vacuum impregnated with glass fibers-epoxy resin insulation. The indirect cooling of the coils through pipes glued to the Al coil casing implies that good thermal contact and low stress interface is achieved between the different interface materials. This requires a huge effort in terms of design and technological development of the techniques for glass-resin impregnation and pre-stressing of such coils.

\section{BARREL TOROID}

The Barrel Toroid [3], Fig. 1, is composed of 8 flat racetrack coils each consisting of two double pancake windings housed in a common aluminium alloy casing that guarantees cooling, rigidity and transfer of the magnetic forces. Every coil has its own vacuum vessel which is connected one to the other through the cryogenic ring for electrical and cryogenic interconnection. 2 X 8 supporting rings complete the warm structure which must guarantee both mechanical stability under static and dynamic loads and a light and open structure. The whole assembly is supported on the feet. Full redundancy is implemented by two cooling circuits at 4.5 $\mathrm{K}$ liquid $\mathrm{He}$ and $60 \mathrm{~K}$ high-pressure $\mathrm{He}$ gas for the cold masses and thermal shields respectively.

Manufacture of the double pancakes consist of winding the conductor with dry glass tape wrapping on a specific mandrel and impregnation in an $\mathrm{Al}$ mould at 2.5 MPa pre-compression. Manufacture of the $\mathrm{Al}$ coil casing is based on connecting long bars, so as to minimize the number of welded connections and to decrease the thickness to be welded. The crucial requirements to be met are the deformation level of such long bars, and the welding quality of such thickness of material. Extensive development and testing of the welding procedure was made by industry on electron beam and TIG/MIG welding. Integration of the impregnated double pancakes into the coil casing is made by gluing with pre-preg layers using the bladder technique to pre-compress the coil. Long Al tubes are inserted between the double pancakes and 
the coil casing filled with glass microballs and impregnated with epoxy resin, then pressurized and cured under a pressure $\mathrm{p}=120$ bar. This gives a safety margin to the shear stress at the interface between the double pancake and the coil casing. Integration of the cold mass and related components into the vacuum vessel requires a large effort in terms of assembly requirements and coordination/responsibility sharing. This operation includes gluing of the cooling pipes onto the cold mass, assembly of the cold mass supports, i.e. the tie rods and the cryogenic stops, assembly of the $\mathrm{Al}$ thermal shields and welding of the cooling pipes to it, assembly of the superinsulation and closing of the vacuum vessel with $160 \mathrm{~m}$ longitudinal welding, Fig. 2.

The model coil B0, with a reduced length of $9 \mathrm{~m}$ and the same cross section, aims to validate the technologies and manufacturing processes of the BT. The fully integrated coil will be delivered to CERN by October 2000 ready for testing. B0 was proven to be fundamental to optimize the production cycle of BT. Following B0 experience many improvements have already been implemented in the winding and impregnation procedures, in the coil casing technology, in the pre-compression technique, in the cold mass support sliding system as well as in the integration scenario and procedure. The production of the coils has started in industry: the first double pancake was successfully impregnated at ANSALDO, I and the vacuum vessel production has started at Felguera CM, E.

\section{END CAP TOROIDS}

The two End Cap Toroids [4], Fig. 3, are inserted in the Barrel Toroid at each end of the Central Solenoid.

Each ECT contains 8 racetrack coils mounted as a single cold mass in a vacuum vessel. Each coil module consists of 2 double pancake windings mounted in an Al coil casing. Impregnation of each coil is done in autoclave with a resin system developed by RAL with high toughness at 4.5 K. An Al castellated thermal shield with its $60 \mathrm{~K} \mathrm{He}$ gas cooling circuit and the superinsulation layers surround the cold mass. All services are connected through a single 
service turret mounted on top of the vacuum vessel. Each ECT is mounted on the main rail structure to allow movement for access to the inner part of the detector. The electromagnetic axial force of about 240 tons pulling each ECT towards the center is reacted at 8 force transfer supports against the $8 \mathrm{BT}$ vacuum vessels.

Production of the cold masses and vacuum vessels is in good progress at HMA Power Systems (Nl) and Royal Schelde Exotech (Nl) and first delivery to CERN is planned in the year 2001 ready for integration and testing.

\section{CENTRAL SOLENOID}

The Central Solenoid [5] with a length of $5.3 \mathrm{~m}$ and a bore of $2.5 \mathrm{~m}$, Fig. 4, provides $2 \mathrm{~T}$ axial magnetic field in the central tracking volume of the ATLAS detector. The CS is installed in a common cryostat with the Liquid Argon Calorimeter in order to minimize the amount of material. For further improvement high-strength aluminium stabilized superconductor was developed by Furukawa and Hitachi in collaboration with KEK. A yield strength $\sigma_{\mathrm{y}}=110 \mathrm{MPa}$ at $4.2 \mathrm{~K}$ and $\mathrm{RRR}=592$ were achieved by using an additive of Ni (1000-ppm) in combination with a cold work of $21 \%$. The support cylinder made of Al alloy $12 \mathrm{~mm}$ thick supports the single layer coil winding inside and is connected to the He cooling circuit outside. The superconducting bus bars and cryo-lines connect the coil through a 10-m long chimney to the proximity cryogenics positioned on top of the detector.

The CS is completed and will be tested at Toshiba at the end of the year 2000. The proximity cryogenics is also completed and has been successfully tested at Toshiba. Thereafter the coil will be delivered to CERN for integration with the Liquid Argon cryostat and final testing before installation.

\section{THE SERVICES}

The cryogenic system consists of: 
1. Internal cryogenics: the cooling circuit of the cold mass and thermal shield, with full redundancy;

2. Proximity cryogenics: the auxiliary cryogenic equipment necessary for the operation of each magnet, i.e. the toroids which are cooled as a single cold mass (600 tons) and the CS (6 tons). The toroid system requires $1200 \mathrm{~g} / \mathrm{s}$ of two-phase $\mathrm{He}$ at $4.5 \mathrm{~K}$, which is circulated by means of 2 centrifugal pumps and related distribution unit, while the CS requires only $7 \mathrm{~g} / \mathrm{s}$ of $4.5 \mathrm{~K} \mathrm{He}$ taken directly from the refrigerator.

3. External cryogenics: the He refrigerator plants and related distribution system, which are common to all magnets.

The refrigeration system comprises two cryoplants: the shield refrigerator and the main refrigerator. The first one is used on the one hand for the cool-down phase of magnets between $300 \mathrm{~K}$ and $100 \mathrm{~K}$ with a cooling capacity of $60 \mathrm{~kW}$ provided by a liquid nitrogen/helium heat exchanger and on the other hand, to cope in steady-state with the shield cooling which requires a non-isothermal capacity of $20 \mathrm{~kW}$ between $40 \mathrm{~K}$ and $80 \mathrm{~K}$. The main refrigerator is used for the final cool-down phase from $100 \mathrm{~K}$ to $4.5 \mathrm{~K}$ and the steady-state operation of the magnet cold masses at $4.5 \mathrm{~K}$. This main refrigerator has an equivalent cooling power of $6 \mathrm{~kW}$ at $4.5 \mathrm{~K}$. The design of the cryogenic system is completed and contracts should be placed by the end of this year.

To decrease the complexity of the distribution lines and increase the overall reliability of the system a common electrical and proximity cryogenic system for the toroids is implemented, Fig. 5,6. The toroids circuit comprises the $21000 \mathrm{~A}-16 \mathrm{~V}$ power supply, two circuit breakers, some $300 \mathrm{~m}$ bus bars, He cooled current leads and the diode-resistor dump unit connected across the supply/breaker and the coils. The ramp-up time of the Toroids is $2-3$ hours. The CS has a separate $8 \mathrm{kA}$ power supply due to its different current rating.

The Magnet Safety System (MSS) insures the magnet protection in stand alone mode with fast and slow discharge request and the personnel safety. The protection of the coils is based on the internal dump of the stored energy through the use of a dump resistor of low value and the 
initiation of quenches in all coils by means of quench heaters. In the slow and fast discharge modes the stored energy is dissipated respectively in the dump circuit and in the coil itself through the quench heaters. For safety reasons 48 VDC UPS (Uninterrupted Power Supply) is used to guarantee the functionality of the system in any faulty condition. Redundancy is foreseen for safety sensors and channels.

The Magnet Control System (MCS) provides the process control for ensuring in full automatic mode the operating sequences of the magnets and the control, regulation and monitoring of all the functional parameters related to the magnet and to the cryogenic, electrical and vacuum systems. Harmonization and standardization requirements are followed for operational and maintenance easiness. It consists of sensors and actuators, programmable logic controllers and the magnet supervisor. High level communication is through a field-bus while all sensor and actuator connections and safety system are hard-wired.

\section{TESTING AND INSTALLATION}

Performance and acceptance test on surface is performed at CERN before underground installation. For size reasons each BT coil will be tested with a magnetic mirror to simulate the electromagnetic load induced by the entire system. Instead each of the 2 ECT magnets and the CS magnet will be fully assembled and tested on surface and then installed as complete systems in the cavern. The test facility [6] is ready at CERN to receive the first coils. After installation in the cavern, Fig. 7, the complete BT will be tested and then integrated with both the ECTs and CS ready for testing of the whole ATLAS Magnet System.

\section{CONCLUSIONS}

The ATLAS Magnet System comprising a solenoid and three toroids is the largest integrated superconducting magnet ever built. It is under the constructing phase and all main contracts have been signed with industry. Production of the cold masses and vacuum vessels for the toroids are in good progress and the first delivery is planned in the year 2001. The CS is nearly 
completed and will be tested at Toshiba at the end of this year. Integration of the toroids and testing is performed at CERN, starting with the test of the B0 model coil in the beginning of the year 2001. The design of the cryogenic, electrical, control and vacuum systems has been finalised and contracts will be placed soon. The present schedule is compatible with the start of underground installation in summer 2003 and commissioning of the whole system in 2005 . Manufacturing and testing of the coils are crucial steps to demonstrate the feasibility and reliability of the ATLAS Magnet System, which for its exceptional size represents a unique challenge in magnet technology.

\section{ACKNOWLEDGEMENTS}

This work is part of the ATLAS detector construction and is financed by the ATLAS Collaboration, about 145 Institutes in 34 countries. In particular the members of the magnet teams at CEA (F), RAL (UK), KEK (J), INFNLASA (I), NIKHEF (NI) and CERN are acknowledged. 


\section{REFERENCES}

[1] ATLAS Magnet System Technical Design Report - 4 Volumes, CERN document CERN/LHCC/97$18+19+20+21(1997)$.

[2] H H J ten Kate, 'The Superconducting Magnet System for the ATLAS Detector at CERN', Proc. MT-16, Ponte Vedra Beach, Florida, USA, 1999.

[3] A Dael et al., 'Synthesis of Technological Developments for the B0 Model Coil and the ATLAS Barrel Toroid Coils', Proc. MT-16, Ponte Vedra Beach, Florida, USA, 1999.

[4] D E Baynham et al., 'Engineering Status of the Superconducting End Cap Toroid Magnets for the ATLAS Experiment at LHC', Proc. MT-16, Ponte Vedra Beach, Florida, USA, 1999.

[5] A Yamamoto et al., 'Progress in ATLAS Central Solenoid Magnet', Proc. MT-16, Ponte Vedra Beach, Florida, USA, 1999.

[6] P Miele et al., 'Status and Commissioning of the ATLAS Magnet Test Facility at CERN', Proc. ASC 2000, Virginia Beach, Virginia, USA, 2000. 